\title{
Application of Information Retrieval Course in College Students' Graduation Thesis Writing
}

\author{
Jing Xie \\ Qilu University of Technology
}

\begin{abstract}
Keywords: Information retrieval; Information resources; Paper writing; Search path; Retrieval method
\end{abstract}

\begin{abstract}
The purpose of this paper is to instruct the important role of the Information Retrieval Course in college students' graduation thesis. First, the significance and function of information retrieval course are illuminated. Then, one by one, the key points of the Information Retrieval are explained in the process of writing. Finally, the author described how to carry out the information retrieval, including selecting of the search tool, the search way, the retrieval method and so on for the college students' graduation thesis writing. It is the effective support for college student thesis, through the information retrieval to ensure the accuracy of the selected topic, the comprehensive of the collection of materials.
\end{abstract}

Information retrieval course has become an important way to carry out information quality education in colleges and universities. In recent years, the information retrieval course has become a public basic course in many colleges and universities. Whether it is for the school or the ratio of starting courses, it has greatly enhanced students' information awareness and capability. ${ }^{[1]}$ With the rapid development of modern information resources, learners change the habit of reading, more and more information resources are used by college students. Information resources are rich in content, not only the storage of information is large, but also has the advantages of high timeliness and high resource sharing, which is convenient for college students to carry out comprehensive, rapid and accurate literature retrieval.

At present, most colleges and universities set the course as undergraduate elective courses, some colleges and universities set up undergraduate compulsory courses. Especially, it is very important to get information resources effectively, analysis and utilization to the graduate students for project research, thesis. Clarify the status of the course and integrate it into the reform of general education curriculum system in colleges and universities, and as the core curriculum of general education, set up information retrieval compulsory courses for undergraduates and postgraduates so as to enhance the readers' information accomplishment to the maximum extent. [2]

It is worth studying on an important learning task for how to use the information retrieval, how to play a key role to information resources in college students thesis writing. At present, under the background of the information gathering, processing and transmission technology represented by computer, multimedia and network, the information network brings the opportunity and challenge to the traditional thesis writing. The paper writing itself is the process of expressing, exchanging and transmitting information. The ability to collect, process, use and create content directly influences the formation and efficiency of the writing process. Therefore, only to meet the requirements of the information society, have high information literacy, the students can competent and meet the needs of graduation thesis writing. [3]

\section{The Meaning and Role of Information Retrieval Courses}

Document retrieval is the process of collecting, organization, store of knowledge and information, and to search information for users. ${ }^{[4]}$ We can get the documents needed in a large number of disordered literatures efficiently and conveniently through the literature search. The purpose of information retrieval course is to cultivate college students who have strong information awareness and information literacy, improve their self-learning ability, so that they can independently learn richer knowledge besides textbooks. ${ }^{[5]}$ The final purpose of information retrieval course is that the students can learn and master the methods and skills of literature retrieval through the course, 
especially, for the thesis writing.

Beneficial to the Collection of Information. It is the fastest and most convenient way to obtain information through digital resource retrieval on the Internet. Without the limitation of the space and time, you can quickly find the large amounts of relevant literature information, through the search engine and a variety of literature search methods. You also can use advanced search to obtain more accurate information. In addition, many digital resources are free for users to browse or download it that provides a convenient condition for a writer.

Beneficial to Inheritance and Innovation. The modern knowledge is profound, not only a large number of types, but also the interdisciplinary phenomenon is more and more increasing. Writing paper is a new exploration based on the achievements or research progresses made by previous scholars. In a paper writing, all of topic, collecting materials, writing, and finalized can not be separated from information retrieval. In the course we will learn to search and collect a great deal of documents, also we will analyze and master the research contents and trends of the related fields, and find and obtain the innovation points of the thesis.

Beneficial to Saving Time. Mastering the basic knowledge of information retrieval and a skilled use of search tools for information resources, we can use less time to quickly and accurately get the necessary literature information, which can avoid human resources, financial and material waste. Mastering the correct literature search strategy, selecting different search tools, ways and methods, can improve the efficiency of retrieval, Speed up the completion of the paper, finish a higher level of the paper.

Beneficial to the Correct Decision. Information retrieval course is a course that integrates library science, information science, philology, informatics, computer science and so on. The purpose of the course is to teach students how to use modern computer technology to inquire the knowledge. Then based on the analysis of existing information and experience, the best option for writing a paper is selected. Decision-making is for a specific purpose, it penetrates into the whole process of writing the paper. The correct decision-making is supported by literature retrieval

\section{The Application of Information Retrieval in the Thesis Writing}

In general, the composition of a paper includes topics selection, information collection, and the first draft; modify the finalized paper and other steps. In the process of writing, the information retrieval should be made fully, and the following key points should be grasped:

The Establishment of the Topic of a Paper. Selecting the title is to determine the direction of scientific research papers. [6] The first task of writing the paper is to set the topic that should be novel, feasible, and scientific and advanced. The title plays a key role for the success or failure of the paper. First of all, we must know the current research situation in the related fields. Graduation thesis is a comprehensive examination of the professional knowledge for students, so the title should be professional and interested by college students.

Firstly, in order to inherit and draw on the results of previous studies on the basis of further study and research, it is very important to read a large number of relevant literatures by using information retrieval. Then, we can understand the history of the project, the current status and development trends, the current research people or institutions, the research result, the existing problems and future research direction timely and accurately.

The reading material is more mastered, the more useful the revelation received or the valuable evidence obtained from it, the writing idea will be broader and the more perfect. Second, to avoid duplication of research topics. The same or similar issues may be studied by many scholars at the same time. Therefore, it is very important to grasp the current status of research through information retrieval. If we find that similar research has been published or reported, we should think if we modify the topic.

Collection and Collation of the Materials Related to the Thesis. After the initial selection of topics, the collection and collation for a lot of materials should be needed. The writing of the thesis depends on these materials. That is, to collect a large number of relevant information, from which to obtain inspiration and basis to serve their own argument. [7] Through a large number of documents 
and quickly reading, the required materials are selected for preservation. This requires a wide range of materials in the process of reading the relevant literature; to read carefully, with the mind, carefully thinking, record their most interested point, learn to find questions.

If there are many documents are involved in the process of collection, it needs careful analysis and deeply reading to find the blank or the focus of the controversy in the field, so find the innovation of the research project. If you can not find the related literatures or the material is relatively small, then the former did not or rarely discussed this topic, the selected topics are novel, you need to further retrieve the basic theory or expertise of the subjects. If there is no appropriate documentation, you need to consider whether the topic is appropriate, whether the paper topic should be changed.

Writing a High Standard of Thesis. Evaluation of academic papers is based on the content quality, the published time, and whether it is better in the research field. These questions can only be evaluated by comparing and analyzing a big number of literature information. ${ }^{[8]}$ Therefore, in order to write a high-level paper, we must first obtain a large amount of information that meets the requirements of the paper topic, and should start from the following aspects when searching and collecting documents:

(1) Monographs and academic papers related to the subject

(2) The review papers written by a prestigious expert, etc.

(3) The recently published main journal related the project.

(4) The related discussion in the classic literature.

(5) The policy related to the project.

(6) The Statistical data from authoritative department and typical examples related to the project.

(7) In addition, in the collection process, attention should be paid to retrieve the authenticity, novelty and accuracy of literatures.

\section{The Steps of Information Retrieval}

The information retrieval in the modern information resources, first of all is to analyze the requirements of the retrieval to determine the scope of the search. Secondly, to select the appropriate search tools and retrieval methods to determine the search path, build search, and then implement the search, browse the results of the inspection. If the results meet the retrieval needs, then the output of the search results. If it is not meet the search needs, you need to re-analysis, modify the search conditions, adjust the above steps to continue the search until getting the complete, accurate and satisfactory literature. The search expression should be able to accurately reflect the topic of the project content, fit for the selected database search syntax rules. ${ }^{[9]}$ In the search process, the appropriate search tools, ways and methods should be selected:

Select the Search Tools: A variety of search tools (retrieval system and its database) have self-characteristics, should be based on the retrieval of the requirements of the project, select the appropriate search tool. And we should pay attention to select professional counterparts, complete, indexing system.

Normally, the information retrieval tools are follows:

CNKI database, Chinese Science and Technology Periodical Database (VIP), wan fang data knowledge service platform, Chinese Science Citation Database, National Newspaper Index Database, Renin University of China Copy Newspaper Full-text Database.

Commonly Chinese e-books include National Digital Library of China, National Digital Library of Science and Technology, Digital Library of Chinese Higher Education, Digital Library of China, National Science Library of the Chinese Academy of Sciences, Superstar Digital Library, Scholarly Scholar Home digital library, Founder e-books, and books with the text of ancient books such as e-books.

The most popular domestic and foreign academic dissertations are as follows:

Chinese Dissertation Bulletin, CALIS University Dissertation Digest and Full-text Database, International Dissertation Digest, American Dissertation Digest and Full-text Database (PQDD), Digital Library of International Dissertations, Index of British Universities Thesis and so 
on.

Select the Search Ways: There are five main ways to search:

(1) Title approach: The title searching is one of the most convenient and fast way.

(2) Authors' path: According to the author's name to search.

(3) No. Route: Find the documents according to the code of the literature, the index (such as the patent number, the chemical substance registration number, the standard number of the technical standard, and the approval number of the drug).

(4) Classification: The search path is using the literature classification number or classification to meet the ethnic search requirements.

(5) Thematic path: Using the content of the project to search the requirements.

Select a Search Method: The rule for selecting a search method is based on the retrieval condition, requirements, and characteristics.

If the search proposal is to understand the whole situation, the method should be used by time sequence. If you just want to know the current status, trends and other dynamic information, the Time reversal method should be used. In order to save time and improve the efficiency of the search, the available sampling method should be used. The above search methods can be used alternately, complement each other, also known as the comprehensive method.

In the whole information retrieval process, the retrieval topic is the most direct and simple retrieval means and the basic way to achieve the purpose of retrieval, namely, the research trend and the development and change. [11] In addition, it is necessary to gradually develop the personal awareness of information, to improve the obtaining ability of the information. We improve the speed and level of writing to achieve the purpose of learning through searching a large number of high-value paper writing materials.

\section{References}

[1] $\mathrm{Wu}$ Meili. College information retrieval course teaching methods and innovative ideas [J]. Electronic production, 2016 (12): 63.

[2] Liu Min. Research on the Curriculum Reform of Information Literacy Education in Colleges and Universities from the Perspective of Liberal Education - Taking Information Retrieval Course as an Example [J] .Chinese Library Science 2015; 203 (1): 67-70.

[3] Ma Xiaoni,Cai Xiaoyun. The Performance and Countermeasures of information Literacy Deficiency in Higher Vocational Liberal Arts Graduation Thesis Writing. Journal of Nanchang College of Education, 2011; 26 (6): 74-75. [J]

[4] Gao Qiaolin. Medical literature search [M]. Beijing: People's Medical Publishing Press, 2012, first edition: 9.

[5] FENG Yuan-feng. Research on Information Retrieval Course Teaching based on Paper Writing [J] .Technology Information Development and Economy 2015; 25 (18): 125.

[6] Zhang Tianqiao, Li Dongfang. Graduation thesis (design) information retrieval and writing guidelines [M]. Beijing: National Defense Industry Press, 2012, first edition: 30-31.

[7] Song Jianxiang, He Ying. The Library Service for the Process of Graduation Thesis Writing[J]. Journal of Qingdao Vocational and Technical College 2011; 24 (5): 73-76.

[8] Wang Xirong, Han Ling, Zhang Qin. Literature retrieval and paper writing [M]. Shanghai: Shanghai Jiaotong University Press, 2013, fourth edition: i.

[9] Huang Junzuo. Literature retrieval and scientific paper writing [M]. Beijing: China Petrochemical Press, 2013, second edition: 25.

[10] Wang Xirong, Han Ling, Zhang Qin. Literature retrieval and paper writing [M]. Shanghai: Shanghai Jiaotong University Press, 2013, fourth edition: 22.

[11]Zhu Jingfang. Modern Information Retrieval Practical Guide [M]. Beijing: Tsinghua University Press, 2012, second edition: 249. 